\title{
Locating and Correcting Errors in Images
}

\author{
Paulo J. S. G. Ferreira, José M. N. Vieira \\ Dep. Electrónica e Telecomunicações / INESC \\ Universidade de Aveiro \\ 3810 Aveiro PORTUGAL \\ E-mail: $\{$ pjf, vieira\}@inesca.pt
}

\begin{abstract}
Most image interpolation or extrapolation algorithms assume that the locations of the unknown pixels are known. In this paper we attempt to remove this restriction. More precisely, we propose an algorithm for locating the incorrect pixels of an image, assuming only partial knowledge of its Fourier transform. Note that this is a nonlinear problem: the unknown quantities are the positions and values of the (say) $n$ erroneous pixels. We show that the positions can be evaluated in $O\left(n^{2}\right)$ or even $O(n \log n)$ flops by solving a set of $n$ linear equations and computing a FFT. The determination of $n$ is part of the algorithm, whose stability is also briefly discussed. The values of the $n$ incorrect pixels can then be estimated using any of the interpolation methods known.
\end{abstract}

\section{Notation}

The complex $N$-dimensional space, with the usual inner product and norm, is denoted by $\mathbb{C}^{N}$. The conjugate transpose is denoted by *. The Fourier matrix $F$ is the unitary $N \times N$ matrix whose elements $F_{a b}$ are given by

$$
F_{a b}=\frac{1}{\sqrt{N}} e^{-\mathrm{j} \frac{2 \pi}{N} a b},
$$

where $\mathrm{j}$ denotes the imaginary unit. The discrete Fourier transform (DFT) of a signal or image $x$ is denoted by $\hat{x}$.

\section{Outline}

Many of the known image interpolation and extrapolation methods have one common characteristic: the positions of the unknown pixels are assumed to be known. In practice, this is not always the case, a fact that renders these methods of limited practical usefulness under some circumstances.

In this paper we attempt to bridge this gap, and propose an algorithm for locating the positions of the incorrect pixels in an image, assuming only partial knowledge of its Fourier transform. Note that this is a nonlinear problem, in which the unknown quantities are the values and positions of the erroneous pixels.

We show that the positions of $n$ incorrect pixels in a $N \times N$ image can be evaluated in $O\left(n^{2}\right)$ or $O(n \log n)$ time by solving a set of $n$ equations and computing a FFT.

It is not necessary to know $n$ beforehand: the computation of $n$ is part of the algorithm. After locating the position of the incorrect pixels, it remains to interpolate them - any of the known interpolation methods can be in principle used for this purpose.

The stability of the algorithm, which naturally depends on the pattern of the incorrect pixels, is also briefly mentioned.

\section{Related work}

There is a vast literature on image interpolation, extrapolation, and related issues - they include implementation aspects, stability analysis, convergence acceleration, noniterative algorithms, the sampled analog of the problem, convergence criteria, the effect of stabilizing constraints, and much more. See [1-17], among many others.

The problem discussed in this paper is also related to error-control codes: it is known that oversampling [18] is an alternative to error-control coding. A discussion concerning the relations between these two and a few other relevant issues, in the context of signal and image reconstruction, can be found in [19].

\section{Locating the incorrect pixels}

This section describes a simple technique that enables the location of $n$ incorrect pixels in any row or column of a $N \times N$ image (in the following we assume that we are dealing with a row). The constraint upon which the method depends is the vanishing of $2 n$ elements of the DFT of the row. Given a unconstrained row of $N-2 n$ pixels this can readily be accomplished 
by computing its DFT, padding $2 n$ zeros, and computing the inverse DFT.

The set

$$
U=\left\{i_{0}, i_{1}, \ldots i_{n-1}\right\}
$$

is used to describe the positions of the $n$ incorrect pixels in a row $x$ of a $N \times N$ image. We denote by $y$ the observed row, and by $e$ the error

$$
e=x-y,
$$

which coincides with $x$ except for the pixels whose indexes belong to $U$. Thus, $e(k)=0$ for all $k \notin U$. Typically, the cardinal of $U$ is much less than $N$, that is, the density $n / N$ of the incorrect pixels is low.

Consider the polynomial

$$
P(z)=\sum_{i=0}^{n} h_{i} z^{i},
$$

defined by

$$
h_{n}=1, \quad P\left(e^{-\mathrm{j} \frac{2 \pi}{N} i_{m}}\right)=0, \quad(0 \leq m<n) .
$$

Let $\left\{j_{0}, j_{1}, j_{2}, \ldots\right\}$ be a set of distinct integers. Substituting $z=e^{-\mathrm{j} \frac{2 \pi}{N} i_{m}}$, multiplying by $e\left(i_{m}\right) e^{\mathrm{j} \frac{2 \pi}{N} i_{m} j_{\ell}}$ and summing over $m$ leads to

$$
\sum_{k=0}^{n} h_{k} \hat{e}\left(k-j_{\ell}\right)=0
$$

where $\hat{e}=F e$ is the DFT of $e$. The idea is to use these equations to determine the $n$ unknown coefficients $h_{k}$ (recall that $h_{n}=1$ by definition).

When $j_{\ell}=-r+\ell(\ell=0,1, \ldots, n-1)$, only $2 n$ samples of $\hat{e}$ need to be known, and the linear equations will be Toeplitz. If the total number of pixels $N$ of the band-limited row of the image is even, and if $r=N / 2$, the matrix will be Toeplitz and Hermitian, because, for real $e$,

$$
\hat{e}(r+i)=\hat{e}^{*}(-r-i)=\hat{e}^{*}(N-r-i)=\hat{e}^{*}(r-i) .
$$

The linear equations can be brought to the form $A x=$ $b$, with

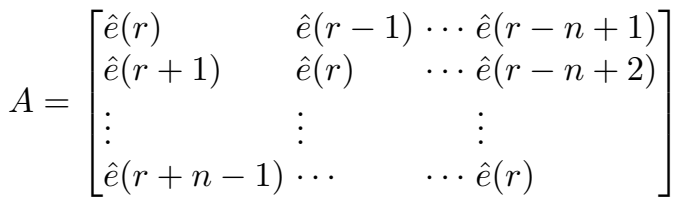

and

$$
b=[\hat{e}(r+1) \hat{e}(r+2) \cdots \hat{e}(r+n)]^{T},
$$

$$
x=\left[\begin{array}{llll}
h_{n-1} & h_{n-2} & \cdots & h_{0}
\end{array}\right]^{T} .
$$

See [20] for details.

In contrast to [18], the matrix is Toeplitz and more importantly, its principal submatrices do not depend on $n$, the number of incorrect pixels. Thus, Levinson's recursion [21] can be used to find the number of errors and simultaneously to solve the system. This costs at most $O\left(n^{2}\right)$ flops (but methods that $\operatorname{cost} O(n \log n)$ also exist).

The zeros of the polynomial $P(z)$ yield the positions of the unknown pixels. The FFT provides a fast and stable way of evaluating the zeros of $P(z)$ (by construction, the zeros are multiples of $\left.e^{-\mathrm{j} \frac{2 \pi}{N}}\right)$.

\section{Applying the method}

The method described in the previous section can be used to locate up to $n$ errors in any row of the image, provided that its DFT contains $2 n$ zeros. If there are more than $n$ errors in a row the method will fail.

It is always possible to pack the image data in a vector (for example, by stacking columns) and then to apply the method to this vector (possibly by dividing it into blocks, and then dealing with each block separately). Although this is possible, it does not necessarily take advantage of the image structure and the natural band-limits that may exist.

Another possibility is to apply the method to the rows and to the columns of the image. In this case, the redundancy will be higher (the columns must also be band-limited, and consequently a $N \times N$ image will contain only $(N-2 n)(N-2 m)$ independent data).

A two-dimensional version of the method used in the previous section is as follows. Consider the product $P(z) Q(w)$, where $P(z)$ and $Q(w)$ are polynomials whose zeros mark the positions of the incorrect pixels, which are now described by a pair of integers $\left(i_{m}, j_{m}\right)$. Thus,

$$
P\left(e^{-\mathrm{j} \frac{2 \pi}{N} i_{m}}\right)=Q\left(e^{-\mathrm{j} \frac{2 \pi}{N} j_{m}}\right)=0, \quad(0 \leq m<n) .
$$

Using an argument similar to the one used in the onedimensional case it is possible to show that, for any $u, v$ belonging to a certain index set,

$$
\sum_{p=0}^{n} \sum_{q=0}^{n} c_{p q} \hat{e}(p-u, q-v)=0
$$

where $\hat{e}$ is the two-dimensional DFT of the error image, and the $c_{p q}$ are the coefficients of the product $P(z) Q(w)$.

If sufficiently many samples of $\hat{e}$ are known these equations can be solved for $c_{p q}$, and the zeros of the 
polynomials found by computing a two-dimensional FFT. The zeros determine the positions of the incorrect pixels, which can then be interpolated using an appropriate algorithm.

\section{Stability}

The condition number of a matrix $M$ with respect to a norm $\|\cdot\|$ is

$$
\kappa(M)=\|M\| \cdot\left\|M^{-1}\right\|,
$$

which, in the spectral norm, becomes

$$
\kappa(M)=\frac{\lambda_{\max }(M)}{\lambda_{\min }(M)},
$$

where $\lambda_{\max }(M)$ and $\lambda_{\min }(M)$ are the largest and smallest eigenvalue of $M$ in absolute value (see [21], for example).

The eigenvalues of a Hermitian $n \times n$ matrix are real. We adopt the convention that they are labeled according to nondecreasing size:

$$
\lambda_{1} \leq \lambda_{2} \leq \cdots \leq \lambda_{n}
$$

The stability of the equations $A x=b$ depends on the $n \times n$ matrix $A$ (1) whose elements are $A_{a b}=$ $\hat{e}(r+a-b)$, with $r=N / 2$. As we have seen, $A$ is Toeplitz and Hermitian if the errors $e$ are real.

There is one important characteristic of the problem: the matrix $A$ can be positive definite, negative definite, or indefinite, depending on the sign of the $e\left(i_{m}\right)$ and the parity of $i_{m}$. It is easy to show, considering the quadratic form

$$
v^{*} A v=\sum_{a=0}^{n-1} \sum_{b=0}^{n-1} A_{a b} v^{*}(a) v(b),
$$

that $A$ is positive definite if and only if $e\left(i_{m}\right)(-1)^{i_{m}}>$ 0 for all $m=0,1, \ldots, n-1$, and negative definite if and only if $e\left(i_{m}\right)(-1)^{i_{m}}<0$ for all $m=0,1, \ldots, n-1$.

Denote by $W$ the diagonal $n \times n$ matrix whose main diagonal elements are

$$
W_{k k}=\sqrt{N} e\left(i_{k}\right)(-1)^{i_{k}} .
$$

Without loss of generality, let $W$ be nonsingular. Introduce the matrix $E$, also $n \times n$, and such that

$$
E_{p q}=\frac{1}{\sqrt{N}} e^{-\mathrm{j} \frac{2 \pi}{N} i_{p} q} .
$$

The role played by these matrices is explained by the following observation: the matrix $A$ is *-congruent to the diagonal matrix $W$, or, more precisely,

$$
A=E^{*} W E \text {. }
$$

This can be used to show that the eigenvalues of $A$ and those of the diagonal matrix $W$ satisfy

$$
\lambda_{\min }\left(E^{*} E\right) \lambda_{k}(W) \leq \lambda_{k}(A) \leq \lambda_{\max }\left(E^{*} E\right) \lambda_{k}(W) .
$$

The extreme eigenvalues of $W$ are of course

$$
e_{\max }=\max _{k}\left|e\left(i_{k}\right)\right|, \quad e_{\min }=\min _{k}\left|e\left(i_{k}\right)\right| .
$$

Then, the condition number of $A$, denoted by $\kappa(A)$, satisfies

$$
\kappa(A) \leq \frac{e_{\max }}{e_{\min }} \kappa\left(E^{*} E\right) .
$$

The eigenvalues of $E E^{*}$ (hence, $E^{*} E$ ) have been studied before because these matrices plays an important role in the band-limited interpolation problems in which the positions of the pixels to be interpolated are known). See, for example, [17,22-24] and the review in [25].

The congruence between $A$ and $W$ reduces the study of the eigenvalues of $A$ to those of $E E^{*}$, because $W$ is diagonal. In one word, we succeeded in reducing the study of the stability problem to two separate, decoupled problems: the eigenvalues of $E E^{*}$, which depend upon the position of the incorrect pixels, but not on the amplitude of the errors; and those of $W$, that depend on the amplitude of the errors, but not on their positions.

\section{References}

[1] A. Papoulis, "A new algorithm in spectral analysis and band-limited extrapolation", IEEE Trans. Circuits Syst., vol. 22, no. 9, pp. 735-742, Sept. 1975.

[2] R. W. Gerchberg, "Super-resolution through error energy reduction", Optica Acta, vol. 21, no. 9, pp. 709-720, 1974.

[3] A. K. Jain and S. Ranganath, "Extrapolation algorithms for discrete signals with application in spectral estimation", IEEE Trans. Acoust. Speech Signal Processing, vol. 29, no. 4, pp. 830-845, Aug. 1981.

[4] J. A. Cadzow, "An extrapolation procedure for bandlimited signals", IEEE Trans. Acoust. Speech Signal Processing, vol. 27, no. 1, pp. 4-12, Feb. 1979.

[5] W. Y. Xu and C. Chamzas, "On the extrapolation of band-limited functions with energy constraints", IEEE Trans. Acoust. Speech Signal Processing, vol. 31, no. 5, pp. 1222-1234, Oct. 1983.

[6] H. J. Trussel, "Convergence criteria for iterative restoration methods", IEEE Trans. Acoust. Speech Signal Processing, vol. 31, no. 1, pp. 129-136, Feb. 1983.

[7] J. L. C. Sanz and T. S. Huang, "Discrete and continuous band-limited signal extrapolation", IEEE Trans. Acoust. Speech Signal Processing, vol. 31, no. 5, pp. 1276-1285, Oct. 1983. 
[8] J. L. C. Sanz and T. S. Huang, "Some aspects of bandlimited signal extrapolation: Models, discrete approximations, and noise", IEEE Trans. Acoust. Speech Signal Processing, vol. 31, no. 6, pp. 1492-1501, Dec. 1983.

[9] C. C. Chamzas and W. Y. Xu, "An improved version of Papoulis-Gerchberg algorithm on band-limited extrapolation", IEEE Trans. Acoust. Speech Signal Processing, vol. 32, no. 2, pp. 437-440, Apr. 1984.

[10] C.-L. Yeh and R. T. Chin, "Error analysis of a class of constrained iterative restoration algorithms", IEEE Trans. Acoust. Speech Signal Processing, vol. 33, no. 6, pp. 1593-1598, Dec. 1985.

[11] S. Singh, S. N. Tandon, and H. M. Gupta, "An iterative restoration technique", Sig. Proc., vol. 11, pp. $1-11,1986$.

[12] F. A. Marvasti, C. Liu, and G. Adams, "Analysis and recovery of multidimensional signals from irregular samples using nonlinear and iterative techniques", Sig. Proc., vol. 36, pp. 13-30, 1993.

[13] T. Strohmer, "On discrete band-limited signal extrapolation", in Mathematical Analysis, Wavelets, and Signal Processing, M. E. H. Ismail, M. Z. Nashed, A. I. Zayed, and A. F. Ghaleb, Eds., Providence, Rhode Island, 1995, vol. 190 of Contemporary Mathematics, pp. 323-337, American Mathematical Society.

[14] P. J. S. G. Ferreira, "Interpolation and the discrete Papoulis-Gerchberg algorithm", IEEE Trans. Signal Processing, vol. 42, no. 10, pp. 2596-2606, Oct. 1994.

[15] P. J. S. G. Ferreira, "Noniterative and faster iterative methods for interpolation and extrapolation", IEEE Trans. Signal Processing, vol. 42, no. 11, pp. 32783282, Nov. 1994.

[16] P. J. S. G. Ferreira, "The stability of a procedure for the recovery of lost samples in band-limited signals", Sig. Proc., vol. 40, no. 3, pp. 195-205, Dec. 1994.

[17] P. J. S. G. Ferreira, "Interpolation in the time and frequency domains", IEEE Sig. Proc. Letters, vol. 3, no. 6, pp. 176-178, June 1996.

[18] F. A. Marvasti and M. Nafie, "Sampling theorem: A unified outlook on information theory, block and convolutional codes", IEICE Trans. Fundam. Electron. Commun. Comput. Sci., vol. E76-A, no. 9, pp. 1383-1391, Sept. 1993.

[19] J. M. N. Vieira and P. J. S. G. Ferreira, "Interpolation, spectrum analysis, error-control coding, and fault-tolerant computing", in Proceedings of the IEEE International Conference on Acoustics, Speech and Signal Processing, ICASSP 97, Munich, Germany, Apr. 1997, vol. III, pp. 1831-1834.

[20] J. M. N. Vieira and P. J. S. G. Ferreira, "Noniterative reconstruction of band-limited signals and coding techniques", in Proceedings of the 7th IEEE Digital
Signal Processing Workshop, DSPW-96, Loen, Norway, Sept. 1996, pp. 149-152.

[21] G. H. Golub and C. F. Van Loan, Matrix Computations, The Johns Hopkins University Press, Baltimore, 1989.

[22] P. J. S. G. Ferreira, "The duality of two recent image interpolation methods", in Proceedings of the Third IEEE International Conference on Image Processing, ICIP-96, Lausanne, Switzerland, Sept. 1996, pp. 735738.

[23] P. J. S. G. Ferreira, "The stability of certain image restoration problems: quantitative results", in Proceedings of the Second IEEE International Conference on Image Processing, ICIP-95, Washington, D.C., U.S.A., Oct. 1995, vol. II, pp. 29-32.

[24] P. J. S. G. Ferreira and A. J. Pinho, "Errorless restoration algorithms for band-limited images", in Proceedings of the First IEEE International Conference on Image Processing, ICIP-94, Austin, TX, U.S.A., Nov. 1994, vol. III, pp. 157-161.

[25] P. J. S. G. Ferreira, "A class of eigenvalue problems in interpolation, extrapolation and sampling", in SampTA'95, 1995 Workshop on Sampling Theory and Applications, Jurmala, Latvia, Sept. 1995, pp. 125-136. 